\title{
ANZEIGER
}

DER

\section{FINNISCH-UGRISCHEN FORSCHUNGEN}

\section{Besprechungen.}

IsRaEL RuoNg, Lappische Verbalableitung dargestellt auf Grundlage des Pitelappischen. Inaugural-Dissertation. Uppsala Universitets Årsskrift 1943: 10. Uppsala 1943. IX + $397 \mathrm{~S}$.

Gustav HasselbRIXK, Vilhelminalapskans ljudlära med särskild hänsyn till första stavelsens vokaler. Akademisk avhandling. Uppsala 1944. XX $+248 \mathrm{~S}$.

Die lappische Sprachforschung hat in Schweden in den letzten Jahren manche wertvolle Ergebnisse gezeitigt, von denen uns dieses Mal zwei akademische Doktorabhandlungen zur Besprechung vorliegen.

Dr. Ruongs Werk ist die erste eingehende Untersuchung aus dem Gebiete der lappischen Wortbildungslehre. Wenn sie sich auch streng auf das Pitelappische beschränkt, sodass alle Vergleiche mit den anderen Dialekten unberücksichtigt gelassen sind, so sind doch die gewonnenen Ergebnisse zum grössten Teil für die Verbalableitung der ganzen lappischen Sprachgruppe gültig.

Zu Beginn sind S. VII - IX einige der interessantesten lautlichen Besonderheiten angeführt, und weiter ist auch die in dem Werke befolgte Schreibweise erklärt. Diese stimmt im wesentlichen mit der Orthographie überein, die Konrad Nielsen in seinen Werken über das Norwegisch-lappische gebraucht hat. Doch sind auch manche Sonderentwicklungen des Pitelappischen in der Orthographie berücksichtigt, auf dem Gebiete des Vokalismus z. B. (in der ersten Silbe) $o a>o$, die Verschmelzung von $a$ und $i e$ zum Wechsel von $a \sim i e$ und die unter bestimmten Bedingungen vor sich gegangenen Veränderungen von $\hat{a}>i, o>u$ und $a>x$; (in der zweiten Silbe) $\hat{a}>0$ nach dem $\varrho$ der ersten Silbe. Es fällt auf, dass 
andererseits aus rein etymologischen Gründen ein Unterschied zwischen dunklem und hellem a gemacht ist. Der Verfasser gibt nämlich an: »Der qualitative Unterschied zwischen den mit $\hat{a}$ und $a$ bezeichneten Lauten ist nicht so gross, dass zwei verschiedene Zeichen nötig wären" (S. IX). Bei der Lautschrift der Konsonanten ist vielleicht die augenfälligste $\mathrm{Ab}$ weichung von dem Nielsenschen System die Berücksichtigung des als Vertreter eines stimmlosen Vokals erscheinenden $h$ als Anfangskomponente von inlautenden, auf einen Tenuisverschlusslaut oder eine Tenuisaffrikata ausgehenden Verbindungen, z.B. $h k, h^{\prime} k$ statt $k k, k 1 k$ usw. Die wortanlautenden unaspirierten Verschlusslaute werden gemäss der norwegisch-lappischen Orthographie transkribiert: mit $d, g, b$, obwohl man offenbar ebenso gut hätte (wie in der Normalorthographie des Lulelappischen) $t, k, p$ anwenden können. Dr. Ruong sagt von diesen Lauten: "Sie . . müssen als tonlose, auf der Grenze zu den Tenues stehende Mediæ betrachtet werden. In meinen Aufzeichnungen habe ich sie mit $D_{t}$ oder $t_{D}, G_{k}$ oder $k_{G}, B_{p}$ oder $p_{B}$ wiedergegeben” (S. VII). Die wortanlautenden Affrikaten, in denen nach dem Verfasser ebenfalls eine schwache Nuancierung zur Media hin ( $t_{D} s$ resp. $\left.t_{D}\right)$ zu vernehmen ist, sind mit $c$ resp. $c$ wiedergegeben, aber im Inlaut ist das Zeichen für dieselben Affrikaten $z$ bzw. $\dot{z}$ (S. IX). Dies ist rom prinzipiellen Standpunkte gesehen natürlich eine Art Inkonsequenz, aber es ist zu verstehen, dass sie nicht vermieden werden konnte.

Die Untersuchung ist teils eine semologische, teils eine morphologische. In ihrem ersten Teil (S. 1-77) berichtet dr. Ruong, zu einem erheblichen Teil auf Adolf Noreen gestützt, über die Bedeutungskategorien und andere damit verbundene Auffassungen. Die klar geschriebene Darstellung ist fest auf das lappische Material gebaut und erhellt, tief eindringend, die Mannigfaltigkeit des verbalen Derivatensystems des Pitelappischen und seine ungewöhnlich feinen Bedeutungsnuancen. Eine Auffassung von den zur Sprache gebrachten Problemen geben die íberschriften der verschie denen Kapitel: "Allgemeines über Bedeutungsgegensätze und ihren morphologischen Ausdruck", "Bedeu1 ungskomplexe und Bedeutungsklassen", "Näheres über die Hauptkategorien", "Die Verbalaktivität bei verschiedenen Arten von Bedeu tungskomplexion", "Passivum, Initiva und Kausativum", "Die Beziehung zwischen den Bedeutungsklassen hinsichtlich der Verbalaktivität", "Systematisches Verzeichnis der wichtigsten Gegensatzkategorien». Der Verfasser hat das geschickte Verfahren angewandt, die verbalen Bedeutungskategorien 
durch algebraische Zeichen darzustellen: $\mathbf{A}=$ intransitiv, $\mathrm{B}=$ transitiv $; \quad \mathrm{I}=$ inaktiv (und passiv), $\mathrm{II}=\operatorname{aktiv} ; \mathbf{1}=$ kursiver Aspekt, 2 = mutativer Aspekt; $\mathbf{a}=$ durativ, $\mathbf{b}=$ frequentativ, $c=$ momentan, usw. (vgl. S. 21). Durch Verbindungen dieser Zeichen kann er mit einer kurzen Formel lange Definitionen zum Ausdruck bringen. So zeigt z. B. die die -adit-Ableitungen charakterisierende semologische Formel AII $(\sim \mathrm{BII})[2 \mathrm{c} \leftarrow 1 \mathrm{a}(\sim \mathrm{b})]$, dass die betreffenden Verben aktive Intransitiva (bisweilen Transitiva) mit mutativem Aspekt und momentaner Aktionsart, sowie zugleich Derivata von Verben mit kursivem Aspekt und durativer oder frequentativer Aktionsart sind.

Auf die semologische analyse folgt das Kapitel "Zweisilbige Stämme und ihre Einteilung in Bedeutungsklassen" (S. 78-101). Dieses Kapitel ist eines der bedeutendsten des interessanten Werkes, erstens deswegen, weil es wohl ein ziemlich vollständiges Verzeichnis der zweisilbigen Verbstämme des Pitelappischen, nach Stammrokalen und Bedeutungen gruppiert, enthält, und zweitens, weil es wichtige IIinweise auf die Geschichte der erwähnten Stammtypen gibt. Es wird festgestellt, dass alle $e$-Stämme nicht deriviert sind, während ein bemerkenswerter Teil von den $\hat{a}_{-}, \varphi^{-}$und vor allem von den $o$-stämmigen Verben Ableitungen zweisilbiger Nomina sind. Wie aus der übersichtlichen Tabelle $\mathrm{S} .79 \mathrm{klar}$ hervorgeht, gibt es $\hat{a}$-stämmige zweisilbige Verben (mitgerechnet die 0 -stämmigen) im Pitelappischen etwa 380, aber $e$-Stämme nur etwa 160 oder etwa $43 \%$ von der Anzahl der $\hat{a}$-Stämme. Die entsprechende Anzahl im Norwegisch-lappischen beträgt nach den ron dem Unterzeichneten auf Grund des Nielsenschen Wörterbuches durchgeführten Berechnungen etwa $59 \%$. Bei den zweisilbigen $\mathrm{N}$ o m i n a, die ursprüngliche Wurzelstämme sind, ist das Verhältnis im Norwegisehlappischen - und zweifellos auch im Pitelappischen - umgekehrt: die e-Stämme sind viel zahlreicher als die $\hat{a}$-Stämme, die etwa $63 \%$ von der Anzahl jener ausmachen. Unter den zweisilbigen $\hat{a}$-Stämmen des Norwegisch-lappischen sind die Gruppen der Nomina und der Verben ungefähr gleich gross, aber unter den $e$-Stämmen bilden die Nomina die grosse Mehrheit; Verben gibt es nur $32 \%$ von der Anzahl der Nomina. Es ist interessant zu sehen, wie diese Verhältnisse im ältesten lappischen Wortschatz liegen, mit anderen Worten bei den zweisilbigen Stänmen, die nachweislich vorlappischen Ursprungs sind. Es zeigt sich, dass das Verhältnis zwischen Verben und Nomina unter den $e$-stämmen genau dasselbe ist wie heutzutage im Norwegisch-Lappischen; Verben gibt es 
$33 \%$ von der Anzahl der Nomina. Aber auch unter den ältesten a-Stämmen sind die Nomina in der Mehrheit; die Anzahl der Verben macht $60^{\circ} \%$ von der der Nomina aus. Man sieht also, dass zur Zeit der Sonderentwicklung des Lappischen die Frequenz der $\hat{a}$-stämmigen Verben sehr gewachsen ist. Zum Teil, aber sicher nicht völlig, erklärt sich diese Erscheinung aus dem von dr. Ruong festgestellten Umstand, dass es unter den $\hat{a}$-stämmigen Verben ziemlich viele abgeleitete Verben gibt. Es bleibt der späteren Forschung zu klären, ob die $\mathrm{Zu}$ nahme der $\hat{a}$-stämmigen Verben hauptsächlich von zufälligen "Modeströmungen" herrührt, oder ob der Vokal der zweiten Silbe in den auf lappischem Boden entstandenen $\hat{a}$-Verben von Anfang an eine bestimmte Bedeutungsnuance bekundet hat. Einen guten Ausgangspunkt für solche Forschungen bietet die von dr. Ruong auf $\mathrm{S}$. 80 seines Werkes gegebene Analyse, wie sich die verschiedenen Stammtypen auf die Bedeutungsklassen verteilen.

Das Kapitel über die kontrahierenden Stämme (S. 102117) beginnt den umfangreichsten Teil des Werkes, in dem die Ableitungssilben und die Bedeutungen der mit ihrer Hilfe gebildeten Verben erklärt werden. Die 30 Kapitel über die unkontrahierten Verbalableitungen umfassen die Seiten 118-278. Diesen Teil charakterisiert der Verfasser folgendermassen: "Der morphologische Teil erhielt das Aussehen eines Kataloges, den ich verhältnismässig vollständig zu gestalten suchte. Es ist infolgedessen ein Mittelding zwischen grammatischer Darstellung und Wörterbuch daraus geworden. Dies Verfahren ist auch insofern motiviert, als es mir die Möglichkeit gab, den gesamten, sich aufs Zeitwort beziehenden Stoff vorzulegen, den ich im Laufe meiner Felduntersuchungen gesammelt hatte." (S. 8). Welche ungewöhnliche Menge Arbeit die Sammlung und systematische Ordnung des Materials gefordert hat, versteht jeder. Als Ganzes ist Dr. Ruongs Ableitungskatalog ron gleich auserlesenem $\mathrm{Ni}$ veau, aber wegen des Reichtums des behandelten Wortstoffes noch bedeutend umfangreicher als Konrad Nielsens Darstellung der Verbalableitungen des Norwegisch-lappischen (Lærebok i lappisk I 229-86). Zu einer solchen Präzisionsarbeit hatte der Verfasser auch natürliche Voraussetzungen. Er beherrscht seinen Stoff souverän in des Wortes voller Bedeutung, war er doch noch bis in sein Schulalter ein Lappenjunge aus Arjeplog, der nur seine Muttersprache sprach. Die Sicherheit und Ausdruckskraft, mit der der Verfasser z. B. die launisch verlaufenden Bedeutungsnuancen der Verben deskriptiver Natur schildert, bereitet dem Leser oft ehrliches 
Vergnügen. Wenn man irgendeine Bemerkung machen möchte, dann zunächst über die Art und Weise, auf die - einer alten Tradition folgend - die Ableitungen selber geschrieben werden. Der Lappologe versteht auch unausgesprochen, dass es prinzipiell ungenau ist von den Ableitungen -dit, -lit, -nit, - $\hat{a} l l \hat{a} t,-\hat{a} h^{\prime} t e t,-\hat{s} s^{i} t e t$ usw. zu sprechen, weil -t nicht zur $\mathrm{Ab}$ leitung gehört, sondern Infinitivendung ist. Die Letztere mitzunehmen, ist für ein unvermeidliches Übel gehalten worden, weil im Endungsrokal der dreisilbigen Verbstämme ein so mannigfacher paradigmatischer Wechsel auftritt, dass es rom rein deskriptiven standpunkt gesehen schwer ist $z u$ entscheiden, welcher Vokal in die abstrahierte Ableitung zu schreiben wäre. Es dürfte sich wohl lohnen zu erwägen, ob nicht künftig doch der alte Gebrauch aufzugeben und die Infinitivendung wegzulassen und der Endungsvokal der dreisilbigen Stämme durch irgendein, sei es auch willkürliches Zeichen wiederzugeben wäre, wie das z.B. Bergsland in seiner Grammatik des Roros'schen Dialekts getan hat (eine andere Sache ist, ob das von Bergsland angewendete $\mathrm{x}$-Zeichen $\mathrm{zu}$ empfehlen ist, denn es bestünde Ursache, den Gebrauch dieses Buchstabens auf die Bezeichnung eines willkürlichen Konsonanten zu beschränken).

Das Werk schliesst mit einer Zusammenfassung (S. 279-90), die eine instruktive $\dot{\mathrm{C}}$ bersicht über die gewonnenen Ergebnisse gibt. Sie erleichtert es zugleich dem Leser, sich über die einzelnen Punkte der Untersuchung zu orientieren.

Durch Dr. Ruongs Verdienst hat sich unser Wissen über ein wichtiges Sonderproblem des Lappischen bedeutend erweitert. Sein Werk ist gleichzeitig sowohl eine zuverlässige Untersuchung als auch ein Wörterbuch der Verben eines bis dahin relativ wenig bekannten Dialektes. Den Forschern der lappischen Ableitungslehre wird es ein höchst wichtiges Quellenwerk sein, auch im Hinblick auf historische Untersuchungen, so gewissenhaft auch der Verfasser selbst sich historischer Schlussfolgerungen enthalten hat. Die hier und da verkommenden Druckfehler sind nicht ernsterer Natur.

Ein gutes Beispiel für den Eifer, mit dem heute die im Schwinden begriffenen südlappischen Dialekte erforscht werden, ist Dr. Hasselbrinks Lautlehre des Vilhelminalappischen. Den Vilhelmina-Dialekt, der zur västerbottenlappischen Gruppe des Südlappischen gehört, hat früher nur K. B. Wiklund untersucht. Seine Arbeit stockte jedoch an ihrem 
Anfang, sodass nur weniges über diesen Dialekt in seinen Werken zu finden ist. Dr. Hasselbrinks Untersuchung füllt somit eine wirkliche Lücke aus; sprachlich dem Vilhelminalappischen am nächsten steht von den bisher genauer behandelten Dialekten der ron Tefsen, ron dem Lagercrantz ein Wörterbuch und eine Grammatik veröffentlicht hat.

Auf den Anfangsseiten seines Werkes gibt der Verfasser einen Überblick über die südlappische Dialektgruppe und ihre typischen lautlichen Eigenheiten. Sowohl der Laut- als auch der Formenlehre des Vilhelminalappischen ist eine grosse Variabilität eigen, derzufolge es der Verfasser für möglich hält, dass der Dialekt vielleicht niemals eine besonders feste form gehabt hat (S. 7, rgl. auch S. 8). Wenn damit das gemeint ist, dass in diesem Dialekt neben den lautgesetzlichen Formen von Anfang an in hohem Grade Analogieformen gebraucht worden wären, so wäre dann wohl eigentlich die Schlussfolgerung zu ziehen, dass das Vilhelminalappische aus einer Dialektmischung entstanden wäre. Da jedoch dr. Hasselbrink offenbar sich den Ursprung des von ihm untersuchten Dialekts nicht so denkt, wäre es sicherlich natürlicher gewesen anzunehmen, dass die analogischen Varianten, so alt wie sie in vielen Fällen auch sein können, nach und nach, zu verschiedenen Zeiten in dem Dialekt erschienen sind. Mit Anerkennung ist die von dem Verfasser mit Sachkenntnis geschriebene Beurteilung der das Südlappische behandelnden Untersuchungen, Textpublikationen und Materialsammlungen $\mathrm{S}$. 8-13 zu er. ähnen.

Die eigentliche Untersuchung zerfällt in zwei Teile: »Lautbestand des Vilhelminalappischen" und "Wechsel und Herleitung der Sprachlaute». Bei der Anordnung des Materials in dieser Weise dürfte Collinders Werk "Lautlehre des waldlappischen Dialektes von Gällivare» als Torbild gedient haben, in dem eine gleichartige $Z$ weiteilung auftritt. Im Einzelnen unterscheiden sich jedoch die Werke bedeutend von einander; so richtet sich Collinders Hauptangenmerk auf die Lantwechsel, während in Dr. Hasselbrinks Cntersuchung die Darstellung des Lautbestandes den grössten Teil einnimmt.

Der erste Teil des Werkes, insgesamt etwa 150 Seiten, enthält auch die umfangreichste und detaillierteste beobachtungsphonetische Darstellung, die über einen einzelnen lappischen Dialekt geschrieben worden ist. Das phonetisch besonders nuancenreiche Südlappische ist schon an sich geeignet, das Interesse des Forschers mit Nachdruck auf die lautlichen Erscheinungen zu lenken, aber auch unabhängig davon ist es offenbar Spezialgebiet des Verfassers, phonetische Beobach- 
tungen zu machen und phonetische Fragen zu erörtern. Es ist auch unausgesprochen klar, welchen Nutzen die Forschung von solchen überaus gründlichen und zuverlässig wirkenden Bestimmungen von Lauten hat. Auch die Transkription des Verfassers scheint, ohne dass sie von der allerfeinsten Art ist, die charakteristischen lautlichen Züge des Dialekts gut zum Ausdruck zu bringen. Weniger geglückt ist seine Art, einige diakritische Zeichen in einer von der herkömmlichen Praxis abweichenden Funktion zu gebrauchen. Besonders störend z.B. ist der Gebrauch des '-Zeichens zur Bezeichnung eines anaptyktischen Vokals. An sich gebraucht man im Südlappischen wegen des besonderen Quantitätssystems des Dialekts kein Zeichen für die starke stufe, als welches sich der betreffende kleine Strich in der lappischen Normalorthographie schon eingebürgert hat, aber wenn man Dr. Hasselbrinks Werk in anderen Zusammenhang zitiert, rerursacht eine solche Form wie z. B. bar'kałdâhkkê '(hår)fläta' unbedingt ein Missverständis beim Leser, der die Besonderheit der Transkription des betreffenden Werkes nicht kennt oder sich nicht daran erinnert. Aus eben denselben Gründen kann man es beklagen, dass das Zirkumflex zur Aufgabe erhalten hat, eine enge Kiefernstellung und schlaffe Lippenartikulation anzuzeigen (wie z.B. beim Vokal der dritten und vierten Silbe des oben zitierten Wortes). Weil die herkömmliche Funktion dieses Zeichens in der finnisch-ugrischen Transkription die ist, eine überlange Quantitätsstufe zu bezeichnen, so ist schon das Bezeichnen des dunklen $a$ in der lappischen Normalorthographie mit $\hat{a}$ zunächst für einen Notbehelf zu halten.

Wenn man aus Dr. Hasselbrinks Untersuchung einige gute Details hervorheben wollte, so wären wohl vor anderem die Darstellungen über die Lautquantität (S. $25-29$ der die Vokale, S. 65-72 der die Konsonanten betreffende Teil) sowie die auch prizipiell interessante Dinge behandelnden Ausführungen über die Palatalität und Velarität, bzw. über die Palatalisierung und Velarisierung (S. 37-40 das die Vokale, S. 86 -94 das die Konsonanten betreffende Kapitel) zu erwähnen. Es scheint, dass der Verfasser bei seiner Präzisierung der Begriffe "Palatalisierung" und "Moullierung" beachtenswerte Gesichtspunkte vorzubringen hat. - Bei der Zergliederung seines Stoffes ist der Verfasser in eine schlechthin allzu kleinliche Genauigkeit verfallen, die zur Folge gehabt hat, dass sich die Darstellung in mosaikartige Stücke zersplittert hat (das zeigt auch schon das aussergewöhnlich breit angeschwollene, nämlich 14 Seiten umfassende Inhaltsverzeichnis des Werkes), und dass dieselben Laute an mehreren verschie- 
denen Stellen behandelt worden sind. Ohne die Hilfe des Inhaltsverzeichnisses würde es dem Leser Schwierigkeiten bereiten, ein Gesamtbild zu gewinnen.

Einen Anhang zu dem Teil über den Lautbestand bildet das Kapitel über den Akzent S. 153-58, gute Präzisionsarbeit auch dieses. S. 155 sagt der Verfasser, dass in den ursprünglichen dreisilbigen Phonemen wie ASg guoppe $\hat{e}_{\mathrm{o}} r \hat{e} b$ 'svamp' und $3 \mathrm{Sg}$ Prs gatsliehtê 'bita' niemals ein Nebendruck auf der zweiten Silbe gewesen ist. Dies ist keineswegs sicher. Ganz klar ist andererseits, dass in einem solehen ursprünglich viersilbigen Phonem wie der Inf. gatsliehtit der Nebendruck auf der zweiten Silbe eine verhältnismässig späte Erscheining ist.

In dem letzteren, den Lautwechseln gewidmeten Teil des Werkes steht der Vokalismus der ersten Silbe im Vordergrund. Nachdem der Verfasser S. 173-77 ein klares Bild von der Entstehung des Vokalsystems der lappischen Sprache gegeben hat, beginnt er, charakteristische Erscheinungen des südlappischen zu untersuchen, unter denen die Vertretung der etymologisch kurzen Vokale der ersten Silbe einen zentralen Platz einnimmt. Unter bestimmten Bedingungen sind urlp. $\check{a}, \check{o}, i, u$ im Südlappischen grundsätzlich auf dieselbe Weise vertreten wie auch in anderen lappischen Dialekten, aber bedingungsweise wird hier das $a$ durch $i$, das $\check{o}$ durch $u$, das $i$ durch $i j$ und das $u$ durch $u w$ vertreten. Ungeachtet dessen, dass der Verfasser die Bedingungen des Vorkommens der in Frage stehenden zweifachen Vertretung der Vokale besonders gründlich beleuchtet, zeigt er nicht die evidente Tatsache, dass die Fälle, wo die Vertretung dieser südlappischen Vokale von der in den anderen Dialekten herrschenden abweicht, ein Geschlossenwerden der etymologisch kurzen Vokale wiederspiegeln, das in der erstgenannten Dialektgruppe eingetreten ist. Ein Ergebnis dieses Prozesses sind auch die Veränderungen $i>i j$ und $u>u w$, sodass also nur die im Südlappischen vorkommenden $i j$ - und $u w$-Fälle ganz und gar von den entsprechenden gemeinlappischen Diphthongen zu unterscheiden sind. Am verdienstvollsten ist das Kapitel über die Vokalharmonie S. 218-33, in dem Dr. Hasselbrink u. a. eine wirklich erschöpfende Darstellung von der regressiven Vokalharmonie oder von dem Umlaut gibt, der im Vilhelminalappischen wie in den südlappischen Dialekten überhaupt einen erstaunlich nuancenvollen Variantenreichtum im Vokalismus der ersten Silbe entwickelt hat.

Dr. Hasselbrink ist seinem eigentlichen Beruf nach Theologe und setzt mit seiner Forschertätigkeit die wertvolle Ar- 
beit fort, die die Seelenhirten der Lappengemeinden seit langem zur Förderung der Kenntnis der lappischen Sprache geleistet haben. Die "Vilhelminalapskans ljudlära» ist nicht nur eine Beweisprobe des Interesses, sondern ungeachtet einiger förmlicher Mängel eine sprachwissenschaftliche Gelehrsamkeit zeigende, verdienstvolle und nötige Untersuchung. Wir wünschen ihrem Verfasser genügend Gelegenheit und Interesse an der Arbeit mit der Sprachforschung auch für die Zukunft.

ERKKI ITKONEx.

ERkкi Itkonex, Struktur und Entwicklung der ostlappischen Quantitätssysteme. Mémoires de la Société Finnoougrienne LXXXVIII. Helsinki 1946. XXII + 267 S.

Von den Werken, die auf dem Gebiet der lappischen Sprachforschung in den letzten Jahren erschienen sind, ist eines der bedeutendsten und gewichtigsten das oben erwähnte Werk Prof. Erkki Itkonens. Es bildet die Fortsetzung und Ergänzung zu dem vom selben Verfasser i. J. 1939 veröffentlichten Werk "Der ostlappische vokalismus vom qualitativen standpunkt aus", weil die von ihm beabsichtigte geschichtliche Darstellung des Vokalismus der ostlappischen Mundarten als Ergänzung zu diesem die qualitative Seite behandelnden Werk eine die Quantität der Vokale und deren Entwicklung deutende Untersuchung forderte. Das Quantitätssystem des Lappischen wiederum ist bekanntlich von solcher Art, dass z. B. die Quantität der Vokale der 1. und 2. Silbe und die Quantität der zwischen diesen Silben stehenden Konsonanten von einander abhängig sind. Infolgedessen hat der Verfasser es für zweckmässig gehalten - und hierin ist er sicherlich im Recht gewesen -, verschiedenartige Worttypen als quantitative Ganzheiten zu behandeln. Auf diese Weise gestaltete sich das Werk auch zu einer Geschichte des inlautenden Konsonantismus der ostlappischen Mundarten. Dieselbe Art der Behandlung hat übrigens Prof. Björn Collinder im zweiten und dritten Buch seiner Doktordissertation "Über den finnisch-lappischen quantitätswechsel I" (Uppsala 1929), die die Quantitätsverhältnisse im Inarilappischen und in den russisch-lappischen Dialekten behandeln, angewandt. Man kann sogar fragen, warum Itkonen es unternommen hat, dasselbe Thema zu behandeln wie Collinder, und warum er die quantitative Seite nicht diesem Werk überlassen hat. Anscheinend hat Itkonen jedoch auch diese Seite 